\title{
Ventral Premotor Cortex Lesions disrupt learning of sequential syntactic structures
}

\author{
Bertram Opitz ${ }^{\text {a }}$, Sonja A. Kotz ${ }^{\text {b }}$ \\ ${ }^{a}$ Experimental Neuropsychology Unit, Saarland University \\ Campus A2.4, 66123 Saarbrücken, Germany \\ Phone: +496813026579, Fax: +496813026516 \\ b.opitz@mx.uni-saarland.de \\ ${ }^{\mathrm{b}}$ Max Planck Institute for Cognitive and Brain Sciences
}

Research Group Neurocognition of Rhythm in Communication, Leipzig, Germany

Short title: Ventral Premotor Cortex Lesions disrupt AGL 


\begin{abstract}
Recent fMRI evidence shows differential involvement of the inferior frontal gyrus (IFG) and the ventral premotor cortex (PMv) in syntactic processing. Our main goal is to specify the precise role of the PMv in the processing sequential structures and whether these processes are a necessary prerequisite for the successful acquisition of linguistic syntax. We tested patients with PMv lesions in an artificial grammar (AG) learning task. Compared to matched controls patients exhibited impaired acquisition of the AG. This impairment was more pronounced for local (referring to adjacent elements within an AG string) as for longdistance dependencies (incorporating recursive structures). This effect was paralleled by a selective reduction of the P600 in response to violations of local dependencies. Crucially, the P600 elicited by violations of long-distance dependencies was comparable between groups. Together, behavioral and ERP results indicate a PMv involvement in processing local sequential information.
\end{abstract}

Key words: artificial grammar, language learning, P600, local and hierarchical sequences 


\section{Introduction}

The representation of grammatical knowledge in language is assumed to involve rules (e.g., Chomsky, 1965). Rule systems build a syntactic hierarchy that depends on embedding local sequences within other sequences in either a linear or hierarchical fashion. While linear structures can be fully specified by transition probabilities between neighboring elements, hierarchical structures, as typically found in so-called phrase structure grammars, are built on long-distance dependencies. Recent neuroimaging studies have shown that the processing of local phrase structure dependencies and long-distance dependencies have different neural correlates. Local structural violations elicit activity in the left frontal operculum and the processing of long-distance dependencies activates Broca's area (Friederici, Fiebach, Schlesewsky, Bornkessel \& von Cramon, 2006b; Friederici, Ruschemeyer, Hahne \& Fiebach, 2003; Röder, Stock, Neville, Bien \& Rösler, 2002). Similar results have been obtained when investigating artificial grammar systems. While the posterior part of the inferior frontal gyrus (BA44) seems to play an important role in the processing of long-distance or hierarchical dependencies, the ventral premotor cortex (BA6, PMv) seems to subserve the processing of local dependencies (Friederici, Bahlmann, Heim, Schubotz \& Anwander, 2006a; Opitz \& Friederici, 2007). In a previous fMRI study (Opitz \& Friederici, 2007), participants were trained and subsequently tested in an artificial grammar (a modified version of BROCANTO, Friederici et al., 2002b). Two types of structural dependencies (local vs. longdistance) were contrasted. The local dependency consisted of two neighboring syntactic elements, whereas the long-distance dependency constituted an embedding of a complementizer structure within a local syntactic combination. When contrasting brain activities associated with violations of local and long-distance dependencies against their respective baselines, the left PMv responded to the processing of local dependencies whereas the opercular part of the inferior frontal gyrus (Brocas area, BA 44) showed activation when processing long-distance dependencies. 
Differential processing of local and hierarchical dependencies has also been shown in the electrophysiological (ERP) studies (Bahlmann, Gunter \& Friederici, 2006; Jiang \& Zhou, 2009). One of these studies was carried out on an artificial language, in which two types of artificial grammar systems consisting of meaningless syllable sequences were learned and then tested (Bahlmann et al., 2006). One type of structure formed local transitions while another type was based on long-distance dependencies. Violations of either structure elicited a late positive ERP component. Based on its spatio-temporal characteristics this component closely corresponds to a late positivity called the P600, representing syntactic reanalysis or integration (Kaan \& Swaab, 2003; Hagoort, 2008; Hahne \& Friederici, 1999). The amplitude of the late positivity varied as a function of syntactic hierarchy, and may thus, reflect enhanced integration costs for long-distance as compared to local dependencies. The second study investigated the comprehension of different levels of the hierarchical syntactic structure in Chinese. Violation of both types of dependencies elicited an anteriorly distributed negativity, with a left hemispheric maximum for violations of local dependencies and right hemispheric distribution for hierarchical dependencies. Contrary to the findings by Bahlmann et al (2006) neither type of violation evoked a late positivity. The absence of the P600 effect was interpreted as being due to the absence of explicit task demands. However, both studies report processing differences for local and long-distance dependencies, confirming previous fMRI evidence.

Although all studies reviewed so far suggest that the PMv is involved in the detection of violations of sequential information in a domain-general fashion (see Fiebach \& Schubotz, 2006, for a detailed disscussion), the temporal dynamics of the neuronal modifications during the acquisition of non-motor, perceptual sequences are less clearly understood. Recent studies provided evidence that motorrelated brain areas were activated in the course of learning sequential finger movements (Jenkins, Brooks, Nixon, Frackowiak \& Passingham, 1994; Lafleur, Jackson, Malouin, Richards, Evans \& Doyon, 2002) and visuo-motor associations (Praeg, 
Esslen, Lutz \& Jancke, 2006; Toni, Ramnani, Josephs, Ashburner \& Passingham, 2001). However, this does not imply that learning linguistic sequences relies also on the PMv as does learning motor sequences. Thus, it is not clear whether this cortical region is necessarily involved in the acquisition of local linguistic dependencies.

A second issue that is currently discussed regards the relationship between the processes subserved by BA44 and BA6. On one hand, it is assumed that the PMv matches simple sequential structures of a sentence with an appropriate default template and Broca's area contributes to the generation of hierarchical organization in language, that operates on serially organized representations provided by the adjacent PMv (Fiebach \& Schubotz, 2006). This view implies a gradient of complexity from simple sequential structures processed in the PMv towards complex hierarchical dependencies handled by Broca's area. On the other hand, results from several fMRI experiments suggest an independence of the processes subserved by the two regions. In most experiments activity in either region is observed (Opitz $\&$ Friederici, 2003, 2004) and both regions differentially respond to experimental variables (Musso, Moro, Glauche, Rijntjes, Reichenbach, Büchel \& Weiller, 2003; Tettamanti, Alkadhi, Moro, Perani, Kollias \& Weniger, 2002).

Thus, our main goal is twofold. Firstly, we sought to specify the precise role of $\mathrm{PMv}$ in the acquisition of linguistic sequential structures and, secondly, we were interested whether these processes operate independently in successful grammar learning. For this purpose patients with lesions centered in the PMv were trained on the modified version of BROCANTO (Friederici et al., 2002b; Opitz \& Friederici, 2007), that allowed directly comparing local phrase structure dependencies and hierarchical, long-distance dependencies. Firstly, if the PMv is, indeed, involved in learning sequential information in a domain-general fashion, we expect impaired acquisition of BROCANTO by the patients as compared to a matched control group. Secondly, if these processes subserved by both structures, the PMv and Broca's area operate independently, a selective impairment in learning local dependencies should be observed. In contrast, if the processes mediated by the PMv 
are a necessary prerequisite for successful grammar learning, we also expect the patient's processing of hierarchical structures to be impaired. Lastly, the respective reduction of the P600 is expected, i.e. a selective reduction of the P600 to violations of local dependencies in case of independent processes subserved by the PMv and Broca's area but an equally reduced P600 to violation of both, local and longdistance dependencies when the processing of hierarchical structures depends upon the intact processing of serially organized representations provided by the adjacent PMv.

In order to ascribe possible changes in the P600 component to syntactic processing deficits and not to varying attentional demands, ERPs were also recorded in an visual classification (i.e. oddball) task. It has been previously demonstrated, that patients with focal vascular basal ganglia lesions and patients without basal ganglia lesions both displayed a P300 in a classic non-linguistic oddball paradigm, while no P600 was elicited by morpho-syntactic violations (Frisch, Kotz, von Cramon \& Friederici, 2003) and argument-structure violations (Kotz, Frisch, von Cramon \& Friederici, 2003) in the patients with basal ganglia lesions. This single dissociation between the P600, normally elicited in a broad range of syntactic anomalies, and the P300 indicates that the P600 is not just a P300-like component in the sense that it reflects the same process of detecting a task-relevant and unexpected event. Consequently, by comparing the P300 oddball effect and the P600 effect elicited by violations of linear and hierarchical dependencies in the patients and their age matched controls we are able to examine the extent to which lesions of the PMv has a general effect on ERP components evoked in cognitive tasks or can be ascribed to a specific syntactic processing deficit in these patients.

\section{Methods}

Subjects: Eight chronic patients were invited to participate in this study. Lesion centered in the PMv. The individual patient's case histories are displayed in Table 
1 and the lesion overlap is depicted in Figure 2. In addition, eight healthy controls were tested. They were matched on gender, age $(\mathrm{M} \pm$ S.D.: patients $=53.8 \pm 4.8$, controls $=56.2 \pm 5.7, \mathrm{t}(14)=-.94, \mathrm{p}=.361)$, and education. All participants gave informed consent prior to the experiment in accordance with the declaration of Helsinki. This study was approved by the ethics committee of the medical department at the University of Leipzig.

Materials: Visual Oddball The material in the visual oddball task consisted of 16 differently shaped objects (circle, triangle, etc.). One quarter of the objects, designated as targets, were easily discernible by an opening (round), while remaining $75 \%$ were not.

Artificial Grammar Learning: The stimulus material is based on a modified version of the artificial language BROCANTO (Friederici et al., 2002b; Opitz \& Friederici, 2007). BROCANTO is formed by the universal principles of natural languages (i.e., it consists of different syntactic word categories and defines phrase structure rules). Each sentence of the artificial language, contains three to eight words and represents a subject-verb-[object] structure. A complementizer structure (C) is used that allows the direct comparison of local phrase structure dependencies and hierarchical, long-distance dependencies. The stimulus set consists of 100 correct sentences, half of them including local dependencies and the other half including long distance dependencies. Another 100 sentences contain a syntactic violation: a long distance violation, a word category repetition, or a local phrase structure violation, both composing local violations (see Table 2 for examples).

Experimental Procedure: Participants engaged in two experiments, a visual oddball experiment followed by an AGL experiment. The oddball experiment was conducted in order to estimate unspecific reductions of ERP components in the patients compared to controls. In this experiment subjects were presented with a total of 300 stimuli, including $25 \%$ targets. Stimulus duration was $200 \mathrm{~ms}$ and the ISI was 1200 
$\mathrm{ms}$, resulting in approximately seven minutes of experimental time. This tested the participants ability to maintain attention throughout the entire experiment. Participants were instructed to fixate the center of the screen and to silently count the targets.

With regards to the AGL experiment participants were trained on a modified version of the miniature artificial grammar system BROCANTO. The training procedure was highly similar to that described in Opitz and Friederici $(2004,2007)$. It comprised alternating learning and test blocks. A brief instruction (7 s) started each block. During learning, participants viewed 20 correct sentences for $7 \mathrm{~s}$ each on a computer monitor and were instructed to extract the underlying grammatical rules. During test blocks, participants were presented with 20 new sentences that were either grammatical (half of the sentences) or ungrammatical. Following a fixation cross $(500 \mathrm{~ms})$ each sentence was presented on a word-by-word basis in the center of a computer monitor. The duration of the word presentation was $500 \mathrm{~ms}$ with an interval of $500 \mathrm{~ms}$ between words. Participant's judged the grammaticality of each sentence. Visual feedback was given for each response. This procedure was repeated 10 times with different sentences.

Data Acquisition and Analysis: Electroencephalograms (EEG) were continuously recorded from $29 \mathrm{Ag} / \mathrm{AgCl}$ scalp electrodes positioned at FP1/2, F7/8, F5/6, F3/4, Fz, FT7/8, FC3/4, T7/8, C3/4, Cz, TP7/8, CP5/6, P7/8, P3/4, Pz, O1/2. The EEG from all sites was recorded with reference to the left mastoid electrode. An additional channel was recorded EEG from the right mastoid, allowing the scalp recordings to be re-referenced off-line to linked mastoids. Vertical and horizontal electrooculograms were recorded with additional electrodes located above and below the right eye and outside the outer canthi of both eyes. Inter electrode impedances were kept below $5 \mathrm{k} \Omega$. The EEG was digitized at a sampling rate of $250 \mathrm{~Hz}$ with a band-pass from DC to $40 \mathrm{~Hz}$.

Off-line data processing included a digital high-pass filter set to $0.1 \mathrm{~Hz}(-3 \mathrm{~dB}$ 
cutoff) to eliminate low frequency signal drifts. An automatic rejection criterion (voltage variation of more than $30 \mu \mathrm{V}$ within a $200 \mathrm{~ms}$ sliding time window) was applied to the EOG channels to mark segments contaminated by eye movement artifacts. These recording epochs were corrected using a linear regression approach (Gratton et al., 1983). Furthermore, all channels were scanned manually for additional disturbances.

In the oddball experiment artifact-free epochs ranging from -200 to $1000 \mathrm{~ms}$ with respect to stimulus onset were averaged separately for each participant, and stimulus type, with the $200 \mathrm{~ms}$ prior to stimulus onset serving as the baseline. Based on visual inspection of the grand average waveform, electrode sites, exhibiting the largest effects were pooled to two topographical ROIs: an anterior ROI (Fz, FP1, FP2) and a posterior region $(\mathrm{Cz}, \mathrm{Pz}, \mathrm{O} 1, \mathrm{O} 2)$. Peak latency and mean amplitudes in the time window 350 to $500 \mathrm{~ms}$ was used for the quantification of the ERP effects. A repeated-measure ANOVA was performed with the within subject factor stimulus type (standard vs target) and the between-subject factor group.

In the AGL experiment epochs for the critical incorrect and correct item lasted 1200 ms commencing $200 \mathrm{~ms}$ pre-stimulus baseline. The respective items were averaged only for correct responses, for each participant, and violation type. For statistical analysis, ERPs were quantified at a left hemispheric ROI (F3, FC3, C3, CP5) and a right hemispheric $\mathrm{ROI}(\mathrm{F} 4, \mathrm{FC} 4, \mathrm{C} 4, \mathrm{CP} 6)$ in the time window from 550 to $700 \mathrm{~ms}$ post stimulus onset. Data were analyzed with repeated-measures ANOVA (alpha level $=.05)$. Condition (correct, local violation, hierarchical violation) and hemisphere ( $\mathrm{LH}$ vs $\mathrm{RH}$ ) were treated as within-subject factors while group was considered a between-subject factor. The Greenhouse-Geisser adjustment for nonsphericity was used whenever appropriate and the corrected $\mathrm{p}$ values are reported together with the uncorrected degrees of freedom. 


\section{Results}

Oddball Experiment: Overall neither healthy controls nor patients had any difficulty to detect target stimuli. Out of a total of 75 targets, healthy controls on average detected all 75 targets, and PMv patients detected 73 targets.

As apparent from Figure 1 targets elicited a P3b component in patients and normal controls. This was confirmed by a main effect of stimulus type (target vs. standard) $\left(F_{(1,14)}=62.69, p<.0001\right)$ in an omnibus ANOVA. Neither the main effects of ROI and group nor any interaction involving these factors reached significance (all $p>$.2). The same analysis was carried out for the peak latency. Here no main effects or any interaction reached significance (all $p>.2$ ), indicating that PMv patients like healthy controls elicited a normal P300.

AGL task - behavior: The performance in terms of endorsement rates in the last two blocks of both groups are depicted in Figure 3. As apparent from the figure the endorsement rates for grammatical sentences did not differ between both groups of participants, whereas non-grammatical sentences were endorsed more frequently as grammatical ones by the patients as compared to controls. Crucially, this was especially the case for sentences containing violations of local dependencies. This visual impression was confirmed by an ANOVA with the repeated-measure factor condition (correct, local violation, hierarchical violation) and the between subject factor group. This analysis revealed a main effect of group $\left(F_{(1,14)}=9.91, p<\right.$ $.001)$, a main effect of condition $\left(F_{(2,28)}=22.2, p<.0001\right)$ and a condition by group interaction $\left(F_{(2,28)}=7.47, p<.01\right)$. Planned contrasts comparing the endorsement rates for both violation conditions exhibited a condition by group interaction $\left(F_{(1,14)}=5.73, p<.05\right)$, indicating a different response pattern for both groups. This interaction was caused by higher endorsement rates for hierarchical as compared to local violations (hierarchical violations: .45 vs local violations: .26, $\left.F_{(1,7)}=5.81, p<.05\right)$ in control subjects, whereas patients did not show different endorsement rates for both violation conditions $\left(.71\right.$ vs $\left..75, F_{(1,7)}<1\right)$. 
AGL task - ERPs: The ERP patterns for critical words comparing grammatical violations and grammatically correct conditions are displayed in Figure 3. As can be seen in this figure, both violation conditions elicited more positive going waveforms as compared to correct sentences. This effect was maximal over the right hemisphere in a time interval from around 550 to $700 \mathrm{~ms}$. Moreover, they reveal a striking group difference. A clear amplitude difference between correct and grammatically incorrect sentences can be observed for healthy controls (Fig. 3), whereas a dramatically reduced effect of grammaticality is present for the patient group.

An ANOVA contrasting the ERPs elicited by the critical words in correct sentences and both types of non-grammatical sentences in both hemispheres revealed a main effect of condition $\left(F_{(2,28)}=4.76, p<.05\right)$ and a marginal significant condition by group interaction $\left(F_{(2,28)}=2.73, p<.08\right)$ and a tendency towards significance for the triple interaction condition by hemisphere by group $\left(F_{(2,28)}=2.1, p<.15\right)$. If the analysis was was done for the right hemisphere only a significant main effect of condition $\left(F_{(2,28)}=4.17, p<.05\right)$ was observed that was qualified by a significant condition by group interaction $\left(F_{(2,28)}=4.38, p<.05\right)$. In controls both violation types elicited similar ERP effects (hierarchical violations: $2.12 \mu \mathrm{V}$ vs local violations: $2.93 \mu \mathrm{V}, F_{(1,7)}<1$ ), whereas for patients this effect was only observed for hierarchical violations (hierarchical violations: $1.27 \mu \mathrm{V}$ vs local violations: -0.99 $\left.\mu \mathrm{V}, F_{(1,7)}=2.94, p<.15\right)$. Taken together, we found a selective impairment of $\mathrm{PMv}$ patients in learning local dependencies as indicated by higher endorsement rates for local as compared to hierarchical structures that were accompanied by a reduction of P600 amplitude over the right hemisphere.

\section{Discussion}

Behavioral and electrophysiological measures in patients with lesions of the ventral premotor cortex were used to examine the role of the PMv in the processing of sequential linguistic information. In the present experiment, PMv patients 
were selectively impaired in the processing of local dependencies in an artificial language, while the acquisition of long-distance, hierarchical dependencies is relatively spared. This is in agreement with recent results demonstrating that the ventral premotor cortex is involved in the detection of local ungrammaticalities in naturally existing languages (Friederici et al., 2003; Raettig et al., 2008), as well as artificial languages mimicking natural grammars (Tettamanti et al., 2002). In addition, a pronounced reduction of a late positivity elicited by violations of local dependencies was observed in the patients compared to the healthy controls. This late positivity elicited by violations in linguistic sequences is in line with previous studies on artificial grammar learning (Friederici et al., 2002b), syllable sequences (Bahlmann et al., 2006) or letter sequences (Lelekov-Boissard \& Dominey, 2002). However, as compared to the 'classical' P600, the positivity in the present experiment has an unusual right frontal distribution. As this positivity extends to parietal areas, the late positivity of the present experiment shares the temporal characteristic of the P600, as well as a partial spatial overlap. A frontal distribution of the P600 has been previously observed in a number of studies and has been interpreted as reflecting the processing of more complex and ambiguous structures (Friederici, Hahne \& Saddy, 2002a; Kaan \& Swaab, 2003; Lelekov-Boissard \& Dominey, 2002). Given that in the present experiment, participants had to extract the grammatical rules underlying the presented sentences, it seems conceivable that the violation of both types of dependencies was an unexpected, rather than an ungrammatical event (see Fonteneau \& van der Lely, 2008, for a similar notion). An alternative explanation can be derived from the fact that the performance of our subjects was not sufficiently high to initiate repair processes, despite their capability of detecting the unusual syntactic structure.

Interestingly, in apparent contrast to previous studies (Bahlmann et al., 2006; Jiang \& Zhou, 2009) the present experiment did not reveal any negativity elicited by both violations of local and hierarchical structures. Bahlmann et al. (2006) reported a posteriorly distributed early negativity that was interpreted as a response to ex- 
pectancy violations towards the incoming constituents (i.e., syllables in this case). However, while Bahlmann et al used two different categories of syllables (equivalent to a transition probability between neighboring elements of 50\%) the present study employed six different word categories leading to much lower transition probabilities (maximally $25 \%$ at the critical positions). These different transition probabilities might have caused different participants' expectancies of the upcoming item. Consequently, the violation of expectancies caused by a non-grammatical item may have been less discernible in the present study and, therefore, not reflected as a negativity in the ERP.

Another interesting finding is the patient's performance in the hierarchical condition. Although PMv patients perform better in detecting violations of hierarchical dependencies as compared to violations of local structures, they, nevertheless, exhibit an impairment in this condition as compared to control participants. One possible explanation might be the fact that in some of the patients the PMv lesions extend into the neighboring opercular part of the IFG, i.e. into BA44, thereby causing partial deficits in processing hierarchical dependencies. Alternatively, it is also conceivable that the patient's problems in acquiring hierarchical dependencies (that extend across phrase boundaries) is a byproduct of their problems in processing local dependencies within phrase boundaries. Perruchet and Pacton (2006) have recently argued that the computation of transition probabilities between neighboring elements might lead to the formation of chunks, that are stored as a processing template against which future incoming information is compared. The boundaries of these chunks are defined as the points where the predictability of successive contiguous elements is the lowest. In the case of an artificial language, such as BROCANTO, the chunk boundaries are equivalent to phrase boundaries. According to this view the patients inability to build up local sequences that constitute a particular noun or verb phrase in BROCANTO will also derogate their ability to process hierarchical structures.

In sum, the present data indicate that PMv is crucially involved in the acquisi- 
tion of local dependencies, thereby adding to the evidence that the PMv is a critical brain structure for the processing of perceptual sequences in a domain-general fashion. As the processing of hierarchical structures is also affected in the patients, the present data suggest, that some aspects of the successful acquisition of local dependencies are a prerequisite for successful language acquisition. The exact nature of these aspects remains to be elucidated in future research. 


\section{References}

Bahlmann, J., Gunter, T. C. \& Friederici, A. D. (2006). Hierarchical and linear sequence processing: an electrophysiological exploration of two different grammar types. J Cogn Neurosci, 18(11), 1829-1842.

Chomsky, N. (1965). Aspects of the theory of syntax. Cambridge, MA: MIT Press.

Fiebach, C. J. \& Schubotz, R. I. (2006). Dynamic anticipatory processing of hierarchical sequential events: a common role for broca's area and ventral premotor cortex across domains? Cortex, 42(4), 499-502.

Fonteneau, E. \& van der Lely, H. K. J. (2008). Electrical brain responses in language-impaired children reveal grammar-specific deficits. PLoS One, 3(3), e1832.

Friederici, A. D., Bahlmann, J., Heim, S., Schubotz, R. I. \& Anwander, A. (2006a). The brain differentiates human and non-human grammars: functional localization and structural connectivity. Proc Natl Acad Sci U S A, 103(7), 2458-2463.

Friederici, A. D., Fiebach, C. J., Schlesewsky, M., Bornkessel, I. D. \& von Cramon, D. Y. (2006b). Processing linguistic complexity and grammaticality in the left frontal cortex. Cereb Cortex, 16(12), 1709-1717.

Friederici, A. D., Hahne, A. \& Saddy, D. (2002a). Distinct neurophysiological patterns reflecting aspects of syntactic complexity and syntactic repair. J Psycholinguist Res, 31(1), 45-63.

Friederici, A. D., Ruschemeyer, S.-A., Hahne, A. \& Fiebach, C. J. (2003). The role of left inferior frontal and superior temporal cortex in sentence comprehension: Localizing syntactic and semantic processes. Cerebral Cortex, 13(2), $170-177$.

Friederici, A. D., Steinhauer, K. \& Pfeifer, E. (2002b). Brain signatures of second language acquisition: Evidence challenging the critical period. Proceedings of the National Academy of Sciences of the United States of America, 99, 529534.

Frisch, S., Kotz, S. A., von Cramon, D. Y. \& Friederici, A. D. (2003). Why the 
p600 is not just a p300: the role of the basal ganglia. Clin Neurophysiol, 114(2), 336-340.

Gratton, G., Coles, M. G. \& Donchin, E. (1983). A new method for off-line removal of ocular artifact. Electroencephalogr Clin Neurophysiol, 55(4), 468-484.

Hagoort, P. (2008). The fractionation of spoken language understanding by measuring electrical and magnetic brain signals. Philosophical Transactions of the Royal Society B: Biological Sciences, 363(1493), 1055-1069.

Hahne, A. \& Friederici, A. D. (1999). Electrophysiological evidence for two steps in syntactic analysis: Early automatic and late controlled processes. J Cogn Neurosci, 11, 194-205.

Jenkins, I. H., Brooks, D. J., Nixon, P. D., Frackowiak, R. S. \& Passingham, R. E. (1994). Motor sequence learning: a study with positron emission tomography. J Neurosci, 14(6), 3775-3790.

Jiang, X. \& Zhou, X. (2009). Processing different levels of syntactic hierarchy: an erp study on chinese. Neuropsychologia, 47(5), 1282-1293.

Kaan, E. \& Swaab, T. Y. (2003). Repair, revision, and complexity in syntactic analysis: an electrophysiological differentiation. J Cogn Neurosci, 15(1), 98110.

Kotz, S. A., Frisch, S., von Cramon, D. Y. \& Friederici, A. D. (2003). Syntactic language processing: Erp lesion data on the role of the basal ganglia. J Int Neuropsychol Soc, 9(7), 1053-1060.

Lafleur, M. F., Jackson, P. L., Malouin, F., Richards, C. L., Evans, A. C. \& Doyon, J. (2002). Motor learning produces parallel dynamic functional changes during the execution and imagination of sequential foot movements. Neuroimage, $16(1), 142-157$.

Lelekov-Boissard, T. \& Dominey, P. F. (2002). Human brain potentials reveal similar processing of non-linguistic abstract structure and linguistic syntactic structure. Neurophysiol Clin, 32(1), 72-84.

Musso, M., Moro, A., Glauche, V., Rijntjes, M., Reichenbach, J., Büchel, C. \& Weiller, C. (2003). Broca's area and the language instinct. Nature Neurosci, 
$8,774-781$.

Opitz, B. \& Friederici, A. D. (2003). Interactions of the hippocampal system and the prefontal cortex in learning language-like rules. Neuroimage, 19, 17301737.

Opitz, B. \& Friederici, A. D. (2004). Brain correlates of language learning: The neuronal dissociation of rule-based versus similarity-based learning. J Neurosci, 24, 8436-8440.

Opitz, B. \& Friederici, A. D. (2007). The neural basis of processing sequential and hierarchical syntactic structures. Human Brain Mapping, 28, 585-592.

Perruchet, P. \& Pacton, S. (2006). Implicit learning and statistical learning: one phenomenon, two approaches. Trends Cogn Sci, 10(5), 233-238.

Praeg, E., Esslen, M., Lutz, K. \& Jancke, L. (2006). Neuronal modifications during visuomotor association learning assessed by electric brain tomography. Brain Topogr, 19(1-2), 61-75.

Raettig, T., Friederici, A. D. \& Kotz, S. A. (2008). Zur interaktion von morphosyntax, phrasenstruktur und semantik bei der satzverarbeitung: eine fmrt-studie. In P. Khader, K. Jost, H. Lachnit \& F. Rsler (Eds.), Beitrge zur 50. Tagung experimentell arbeitender Psychologen. Lengerich: Pabst Science.

Röder, B., Stock, O., Neville, H., Bien, S. \& Rösler, F. (2002). Brain activation modulated by the comprehension of normal and pseudo-word sentences of different processing demands: A functional magnetic resonance imaging study. Neuroimage, 15(4), 1003-1014.

Tettamanti, M., Alkadhi, H., Moro, A., Perani, D., Kollias, S. \& Weniger, D. (2002). Neural correlates for the acquisition of natural language syntax. Neuroimage, $17,700-709$.

Toni, I., Ramnani, N., Josephs, O., Ashburner, J. \& Passingham, R. E. (2001). Learning arbitrary visuomotor associations: temporal dynamic of brain activity. Neuroimage, 14(5), 1048-1057. 
Table 1

Patient's history and localization of lesions

\begin{tabular}{|c|c|c|c|c|c|c|c|c|}
\hline No. & Sex & Hand. & $\mathrm{AaT}$ & TsL & Etiology & $\begin{array}{l}\text { Site of } \\
\text { Lesion }\end{array}$ & Lesion Description & $\begin{array}{l}\text { Affected } \\
\text { Brodmann } \\
\text { Areas }\end{array}$ \\
\hline 1 & $\mathrm{f}$ & $\mathrm{R}$ & 47 & 10 & $\begin{array}{l}\text { Angioma, } \\
\text { postoperatively }\end{array}$ & $\mathrm{B}$ & $\begin{array}{l}\text { left MFG, IFG, } \\
\text { IFGPOp,IFGPTr, } \\
\text { aIn, EC; bilateral Th }\end{array}$ & $\begin{array}{l}08094445 \\
46\end{array}$ \\
\hline 2 & $\mathrm{~m}$ & $\mathrm{~L}$ & 67 & 7 & PMI & $\mathrm{L}$ & MFG, IFGPOp, PrG & $\begin{array}{l}06434445 \\
47\end{array}$ \\
\hline 3 & $\mathrm{~m}$ & $\mathrm{R}$ & 32 & 6 & PMI & $\mathrm{L}$ & $\begin{array}{l}\text { IFGPOr, IFGPTr, } \\
\text { IFGPOp, PrG, aIn }\end{array}$ & $\begin{array}{l}06434445 \\
47\end{array}$ \\
\hline 4 & $\mathrm{~m}$ & $\mathrm{R}$ & 39 & 6 & PMI & $\mathrm{L}$ & IFGPOp, PrG & 040644 \\
\hline 5 & $\mathrm{~m}$ & $\mathrm{R}$ & 56 & 5 & $\begin{array}{l}\text { PMI, } \\
\text { noncompressive } \\
\text { temporal arachnoid } \\
\text { cyst }\end{array}$ & $\mathrm{L}$ & $\begin{array}{l}\text { MFG, IFGPOr, } \\
\text { IFGPTr, IFGPOp, } \\
\text { PrG, aIn }\end{array}$ & $\begin{array}{l}06080944 \\
454647\end{array}$ \\
\hline 6 & $\mathrm{f}$ & $\mathrm{R}$ & 51 & 4 & SAH & $\mathrm{B}$ & $\begin{array}{l}\text { right PrG PrG } \\
\text { IFGPOp, left LOrG, } \\
\text { POrG, CC }\end{array}$ & $\begin{array}{l}\text { L } 1147 \text { R } 06 \\
44\end{array}$ \\
\hline 7 & $\mathrm{f}$ & $\mathrm{L}$ & 46 & 6 & BZI & $\mathrm{B}$ & $\begin{array}{l}\text { left PoG, right } \mathrm{PrG} \text {, } \\
\text { bilateral SPL, } \mathrm{Cu}\end{array}$ & $\begin{array}{l}\text { L } 010203 \text { R } \\
0406 \text { B } 07 \\
19\end{array}$ \\
\hline 8 & $\mathrm{~m}$ & $\mathrm{R}$ & 52 & 4 & PMI & $\mathrm{L}$ & $\begin{array}{l}\text { IFGPOr, WM of } \\
\text { IFG, LOrG, WM of } \\
\text { PrG, aIn }\end{array}$ & 061147 \\
\hline
\end{tabular}

Abbreviations: f female; m male; L left; R right; B bilateral; PMI partial middle cerebral artery infarction; SAH subarachnoidal hemorrhage; BZI border-zone infarction; orbital region: MOrG medial orbital gyrus; LOrG lateral orbital gyrus; POrG posterior orbital gyrus; frontal region: IFG inferior frontal gyrus; IFGOr inferior frontal gyrus, orbital part; IFGTr inferior frontal gyrus, triangular part; IFGOp inferior frontal gyrus, opercular part; MFG middle frontal gyrus; SFG superior frontal gyrus; PrG precentral gyrus; occipital region: $\mathrm{Cu}$ Cuneus, subcortical: Th thalamus; $\mathrm{CI}$ internal capsule; EC external capsule; CC corpus callosum. Age and time since lesion (at test) in years. AaT Age at Test; TsL Time since Lesion; Hand. Handedness 
Table 2

Examples of grammatical and non-grammatical sentences of the modified version of BRO-

CANTO

\begin{tabular}{ll}
\hline \hline Grammatical sentences & Nongrammatical sentences \\
\hline
\end{tabular}

Long distance (a) aak gum prez caf aak trul rix (b)* aak gum prez nöri aak trul rix

$\begin{array}{lllllllllllllllll}\text { dependencies } & \mathrm{D} & \mathrm{N} & \mathrm{V} & \mathrm{C} & \mathrm{D} & \mathrm{N} & \mathrm{V} & \mathrm{D} & \mathrm{N} & \mathrm{V} & \mathrm{M} & \mathrm{D} & \mathrm{N} & & V\end{array}$

Local (c) aak plox glif rüfi aak böke gum (d)* aak plox glif pel aak böke gum

$\begin{array}{lllllllllllllllll}\text { dependencies } & \mathrm{D} & \mathrm{N} & \mathrm{V} & \mathrm{M} & \mathrm{D} & \mathrm{A} & \mathrm{N} & \mathrm{D} & \mathrm{N} & \mathrm{V} & V & \mathrm{D} & \mathrm{A} & \mathrm{N}\end{array}$

D - determiner, $\mathrm{N}$ - noun, V - verb, M - verb modifier, A - adjective, C - complementizer

Violations that rendered sentences non-grammatical are in italics. Note that the nongrammatical version of the long distance dependency condition (b) is ungrammatical as the sequence D-N-V at the end of the sentence is only licensed after a C-element as in its grammatical counterpart (a). In case of local dependencies ungrammaticality is realized by two successive elements of the same class (V-elements in the present example), not allowed by the grammar. An example of each of these sentences for English would be the following:

(a) The man wondered whether the boy lied.

(b) *The man wondered slowly the boy lied.

(c) The man greeted enthusiastically the young girl.

(d) *The man greeted saw the young girl. 
Table 3

Mean endorsement rates $( \pm S E M)$ for grammatical sentences, and sentences containing a violation of either local or hierarchical dependencies. Note that patients endorsed nongrammatical sentences more frequently as grammatical as compared to healthy controls.

\begin{tabular}{lcc}
\hline \hline & Control Subjects & Patients \\
\hline correct sentences & $.81(.07)$ & $.80(.04)$ \\
hierarchical violations & $.45(.10)$ & $.71(.05)$ \\
local violations & $.26(.07)$ & $.75(.09)$ \\
\hline \hline
\end{tabular}




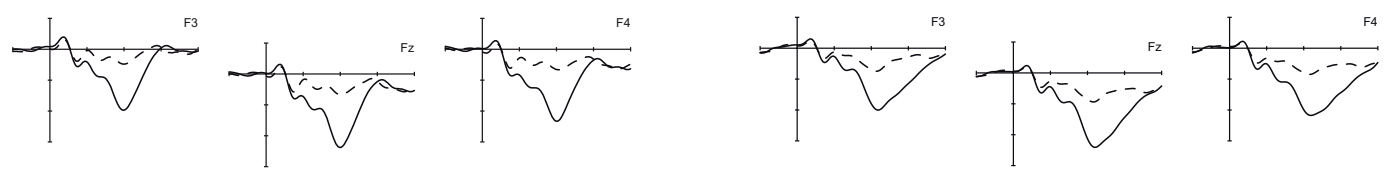

Control Subjects

- - standards $\quad-$ deviants

Patients
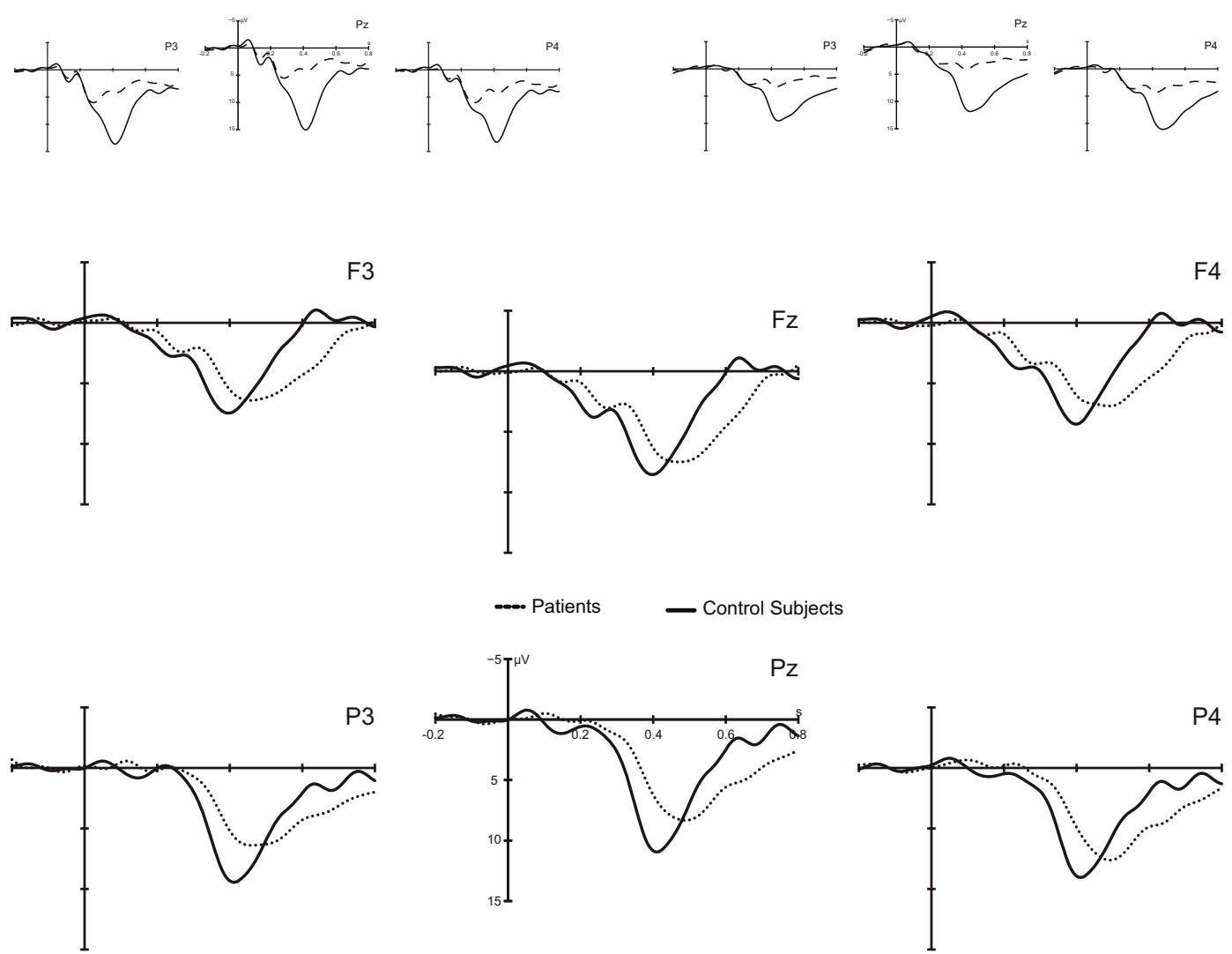

Figure 1. P300 oddball effects in healthy controls (upper left panel) and PMv patients (upper right panel). Dotted lines represent standard stimuli, solid lines the targets. The lower panel depicts the target - standard difference waveform for healthy controls (solid line) and patients (dotted line). 


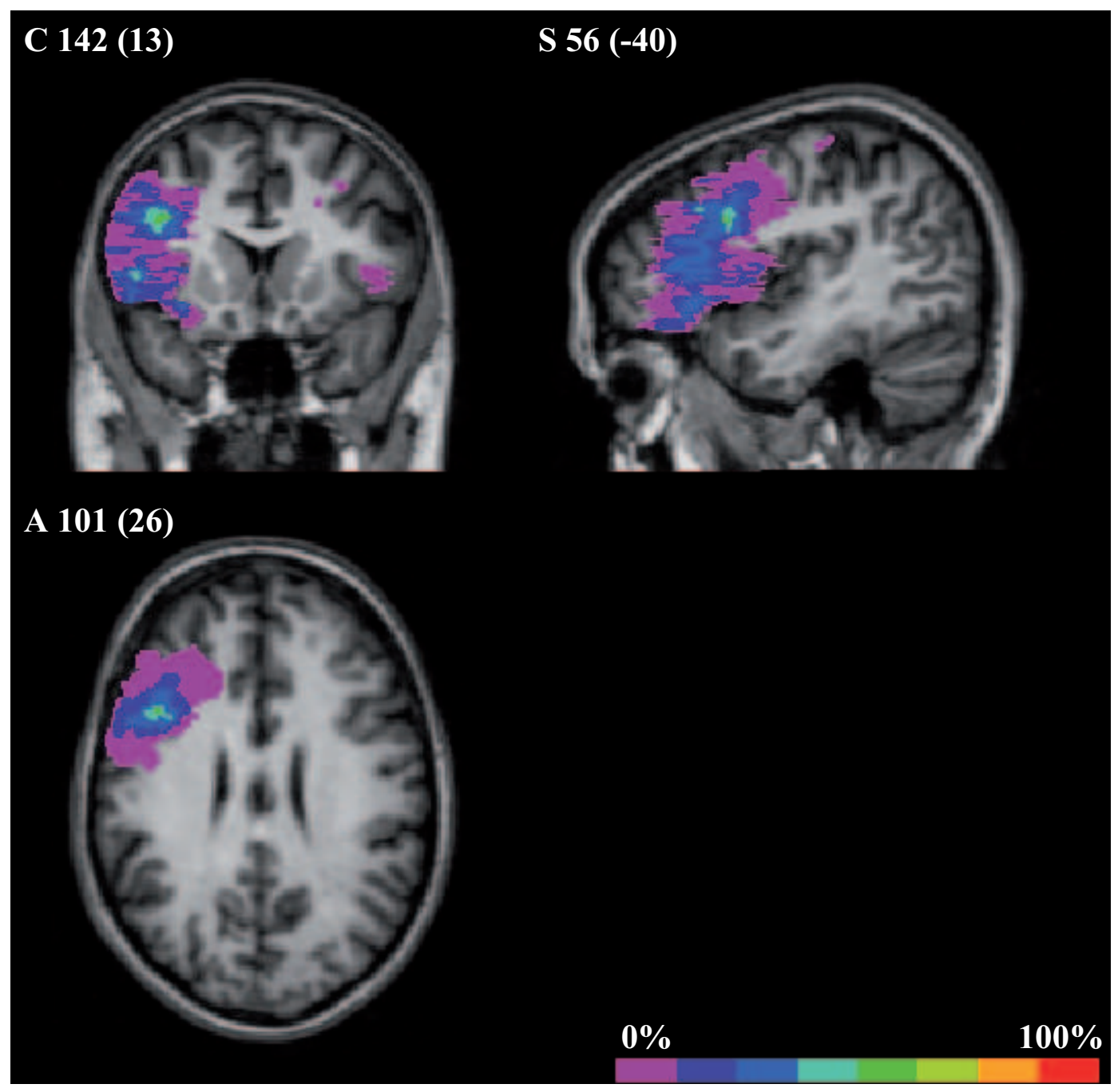

Figure 2. Lesion overlap. Displayed are representative coronal C, sagittal S and axial A slices with corresponding Talairach coordinates in brackets. Regions of interest of the eight patients described in Tab. 1 are overlapped on a reference image (healthy control, female, 23 years of age). In the color bar the colors indicate the number of overlapping ROIs. The leftmost (dark violet) color indicates the index for a single ROI, while the rightmost (bright red) color shows the index for all the ROI's overlapping. Here maximal lesion overlap is found at precentral gyrus at junction with middle frontal gyrus, indicated by turquoise/green shades. 

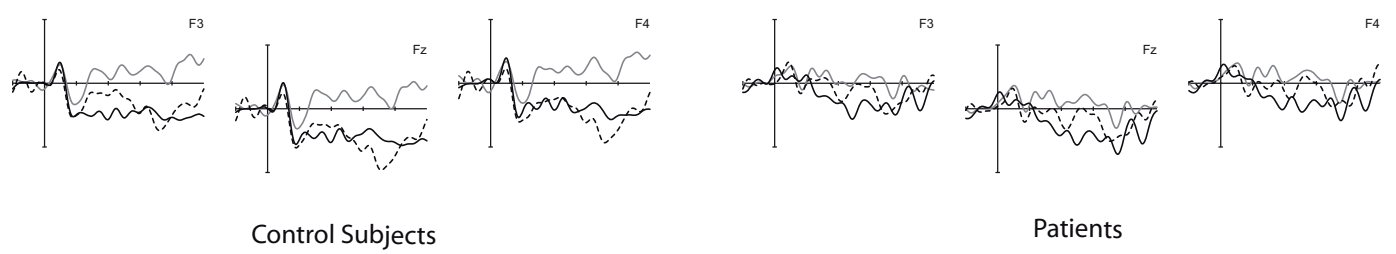

Control Subjects

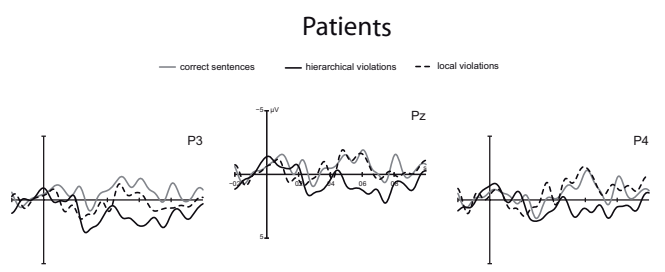

Figure 3. ERPs depicted at selected electrodes for correct sentences (gray), hierarchical violations (solid black) and local violations (dotted). The left panel shows data from healthy controls, the right panel shows patient data. 


\section{Control Subjects}

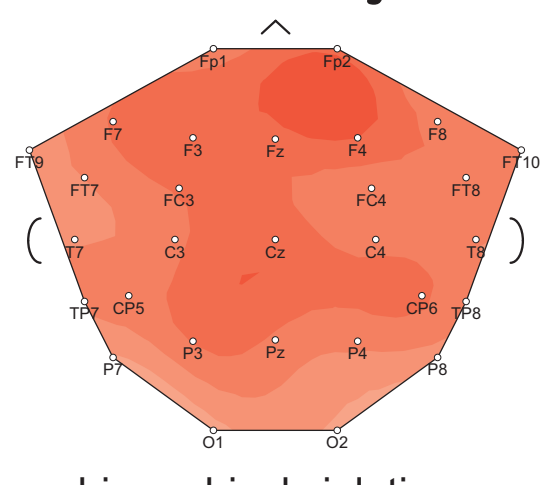

hierarchical violation

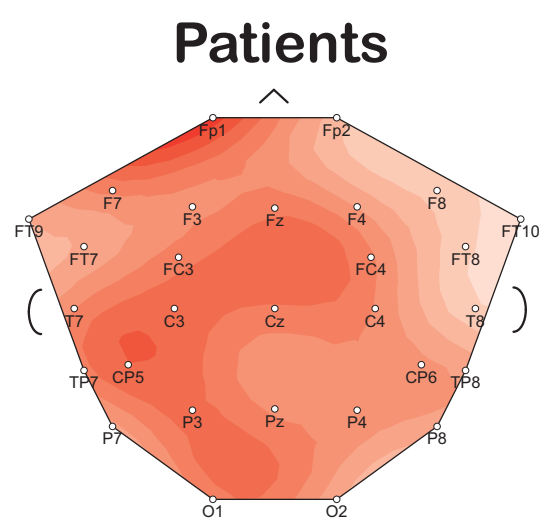

hierarchical violation

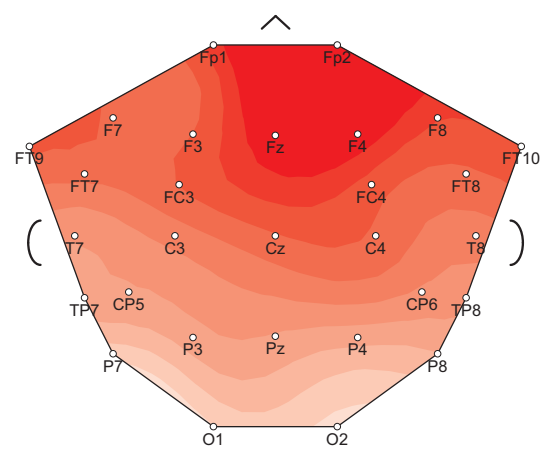

local violation
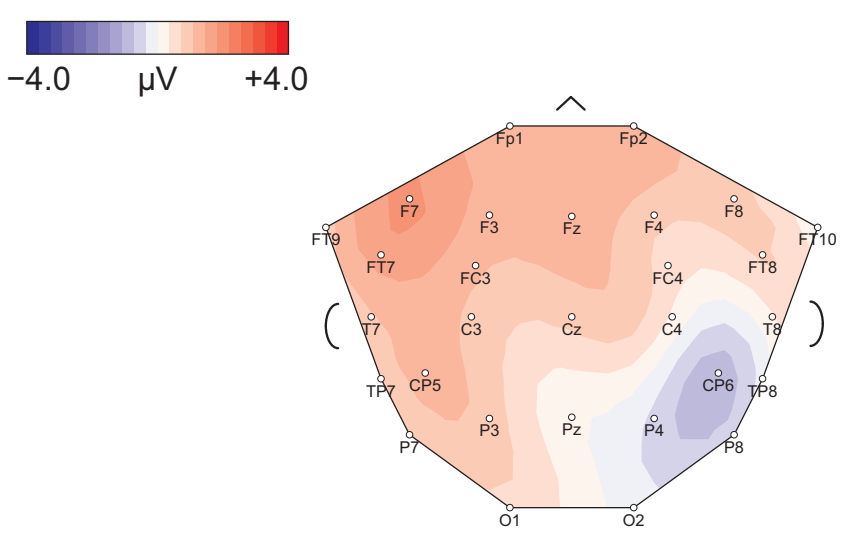

local violation

Figure 4. Scalp potential maps of the difference waveforms (upper panel: hierarchical violation - correct, lower panel: local violation - correct) in the time window from 550 to 700 ms post stimulus onset depicted for healthy controls (left) and patients with PMv lesions (right). Note the selective reduction of the P600 elicited by local violations in the patient group. 